\title{
A New Trick for a Conserved Enzyme: Mevalonate Kinase, a Glycosomal Enzyme, Can Be Secreted by Trypanosoma cruzi and Modulate Cell Invasion and Signaling. Is It Another Moonlighting Enzyme?
}

\author{
Diana Bahia ${ }^{1,2 *}$ \\ 1 Departamento de Microbiologia, Imunologia e Parasitologia, Escola Paulista de Medicina, Universidade Federal de São \\ Paulo, São Paulo, Brazil, ${ }^{2}$ Departamento de Biologia Geral, Instituto de Ciências Biológicas, Universidade Federal de Minas \\ Gerais, Minas Gerais, Brazil
}

Keywords: moonlighting protein, mevalonate kinase, Trypanosoma cruzi, invasion, signaling

\section{INTRODUCTION}

"Moonlighting" means doing a second job, often at night, to earn more money. So-called moonlighting proteins are proteins that play other, generally unusual and unexpected, roles in addition to their canonical roles. They have been described in a wide range of organisms from

OPEN ACCESS

Edited by:

Brice Rotureau,

Institut Pasteur, France

Reviewed by:

Frédéric Bringaud,

Centre National de la Recherche Scientifique (CNRS), France

Najma Rachidi,

Institut Pasteur, France

*Correspondence:

Diana Bahia

dianabahia@hotmail.com

Received: 25 April 2017 Accepted: 15 September 2017 Published: 29 September 2017

Citation:

Bahia D (2017) A New Trick for a Conserved Enzyme: Mevalonate Kinase, a Glycosomal Enzyme, Can Be Secreted by Trypanosoma cruzi and Modulate Cell Invasion and Signaling. Is It Another Moonlighting Enzyme?

Front. Cell. Infect. Microbiol. 7:426. doi: 10.3389/fcimb.2017.00426 bacteria to mammals, and their additional functions vary greatly (reviewed in Collingridge et al., 2010; Huberts and van der Klei, 2010; Henderson and Martin, 2011).

The phenomenon was first described in the well-studied neuropeptides somatostatin and growth hormone-releasing factor (GRF), which were also found to have modulatory effects on the immune system. This second function was later called moonlighting (Campbell and Scanes, 1995). The term was used for the second time in a study of enzymes involved in repair of DNA double-strand breaks that were also involved in maintenance of telomeres (Weaver, 1998). However, the first study to establish the concept of moonlighting proteins was that of Jeffery (1999), who proposed that the key requirement for a protein to be classified as a moonlighting protein is that it have distinct biological functions that are not due to gene fusions, and that it be neither a multiple RNA splice variant nor a protein fragment. The additional function of a moonlighting protein may be attributed to its original active site or to novel sites (reviewed in Huberts and van der Klei, 2010; Henderson and Martin, 2011); however, in enzymes it often depends on the enzyme's catalytic activity and location. In some cases, the protein performs its original function within a cell or cell compartment and a different biological function when it is in a different cell compartment or even when it is secreted (reviewed in Collingridge et al., 2010; Huberts and van der Klei, 2010; Henderson and Martin, 2011). Hence, moonlighting activity is partially dependent on the protein's environment, a phenomenon for which the term "geographical moonlighting" has been proposed. Some moonlighting proteins have been reported to be secreted by cells and to affect intercellular signaling events, while others can act as cell surface receptors or ligands for selected receptors (reviewed in Henderson and Pockley, 2010). In eukaryotes, most proteins that display moonlighting functions are enzymes involved in metabolic processes, such as the glycolytic pathway, a universal metabolic pathway. Indeed, almost every enzyme involved in glycolysis has been reported to moonlight (Gómez-Arreaza et al., 2014), and some have been shown to play important roles in processes such as cell signaling and transformation, DNA repair, transcription, apoptosis, and detoxification, indicating that they also act in distinct subcellular compartments often unrelated 
to their original activity, i.e., glycolysis (Gómez-Arreaza et al., 2014; Erez and DeBerardinis, 2015). For example, GómezArreaza et al. (2014) presented evidence for glycolytic enzymes (e.g., glucose-6-phosphate isomerase) in parasites that exert an unexpected second function.

\section{MOONLIGHTING PROTEINS IN INTRACELLULAR PARASITES}

Numerous moonlighting enzymes normally involved in metabolic pathways in pathogenic protozoa have been reported to display different functions that enable them to act as virulence factors. For example, Plasmodium spp. aldolase has been reported to act as an adhesin, binding to the host cell membrane (Buscaglia et al., 2003). Aldolase has also been shown to interact with actin and surface adhesion molecules, facilitating host cell invasion by Toxoplasma and Plasmodium (Buscaglia et al., 2003; Jewett and Sibley, 2003; Baum et al., 2006).

Plasmodium aldolase connects motor actin filaments to proteins of the thrombospondin-related anonymous protein (TRAP) family, which are transmembrane adhesive proteins, resulting in transduction of motor energy across the parasite's surface. TRAP-aldolase interaction is crucial for host hepatocyte invasion by Plasmodium sporozoites and red blood cell invasion by merozoites (Buscaglia et al., 2003; Jewett and Sibley, 2003; Baum et al., 2006; Bosch et al., 2007). Interestingly, glycolytic and non-glycolytic functions of aldolases cannot occur simultaneously, because adhesins bind to the active site, blocking catalytic activity.

Enolase has been described as a multifaceted protein, and there is strong evidence that it can exert several non-glycolytic functions in pathogens (Karkowska-Kuleta and Kozik, 2014). Knowledge of the stage-specific expression and sub-cellular localization of a protein is helpful in elucidating its moonlighting functions. Enolase has also been found in the nucleus, food vacuole, cytoskeleton, and plasma membrane of Plasmodium falciparum, in addition to its normal location in the cytosol (Bhowmick et al., 2009).

P. falciparum can recruit plasminogen to the ookinete surface via the enolase internal lysine motif, DKSLVK. This process appears to be essential during degradation of the extracellular matrix (glycocalyx) that covers the mosquito midgut epithelium, because it enables the pathogen to complete a complex life cycle within its mosquito vector (reviewed in Karkowska-Kuleta and Kozik, 2014).

In the trypanosomatid Leishmania mexicana, enolase may be found in the cytosol and outer cell membrane as well as in the glycosome where it is normally found, and plays a part in capture of plasminogen by the parasite (Quinones et al., 2007; Vanegas et al., 2007). Hexokinase, which is generally present in glycosomes in the trypanosomatid $L$. donovani, has also been found in the flagellar pocket of the plasma membrane and displays a hemoglobin receptor-like function, suggesting that it is involved in capture of iron or heme (Krishnamurthy et al., 2005).

There are two online moonlighting protein databases: MultitaskProtDB: (wallace.uab.es/multitask) and MoonProt database (www.moonlightingproteins.org/moonlighting.php).
Both contain information about moonlighting proteins for which biological evidence indicates that at least two unrelated functions are carried out by one polypeptide chain. The databases contain details for $>270$ proteins with confirmed moonlighting functions.

Other parasite moonlighting enzymes include Plasmodium dihydrofolate reductase-thymidylate synthase (DHFR-TS), which binds to its cognate mRNA and blocks its own translation, although DHFR-TS mRNA binding does not depend on enzymatic activity (Zhang and Rathod, 2002).

\section{TRYPANOSOMA CRUZI MEVALONATE KINASE (TcMVK) AS A MOONLIGHTING ENZYME}

Mevalonate kinase (MVK), an enzyme involved in synthesis of isoprenoids, is found in a wide range of organisms from bacteria to mammals. The mevalonate pathway is an important metabolic pathway that provides cells with bioactive molecules essential for multiple cellular processes. In the trypanosomatids Trypanosoma brucei and Leishmania major, biosynthesis of sterols is localized to multiple intracellular compartments, and HMG-CoA reductase (from acetyl CoA) and mevalonate are produced mainly in mitochondria, whereas mevalonate phosphorylation occurs only in glycosomes (Carrero-Lerida et al., 2009).

Our group characterized the ergosterol biosynthesis enzyme mevalonate kinase-TcMVK- in T. cruzi (Ferreira et al., 2012, 2016). This enzyme is localized in T. cruzi glycosomes, where it plays its conventional (conserved) role converting mevalonic acid to 5-phosphomevalonic acid (Ferreira et al., 2016). We found unexpectedly that TcMVK is also secreted into extracellular medium, where it binds HeLa cells and modulates T. cruzi cell invasion (Ferreira et al., 2016). Addition of a recombinant form of TcMVK to cultured HeLa cells triggered phosphorylation of MAPK pathway components and proteins involved in actin cytoskeleton changes. We further discovered that the active form of TcMVK is the dimeric oligomeric form, and only fresh purified recombinant TcMVK was capable of adding phosphate to mevalonic acid (Ferreira et al., 2016), suggesting that binding to cell host membrane and modulation of $T$. cruzi cell invasion by TcMVK are not due to its catalytic function. It has been suggested that TcMVK reaches extracellular medium by classical secretory pathways (signal peptide-mediated translocation into the endoplasmic reticulum, and secretion). Additional mechanisms such as extrusion of microvesicles may also be involved (reviewed in Watanabe Costa et al., 2016), but this possibility requires further investigation. TcMVK stimulates and regulates host cell responses to facilitate T. cruzi internalization, suggesting that it functions as a novel T. cruzi virulence factor, and is likely to have other as yet unidentified roles.

By chance, during studies of immune evasion by parasites, my attention was caught by an article entitled "Moonlighting Enzymes in Parasitic Protozoa” (Collingridge et al., 2010). I realized that TcMVK can be considered another moonlighting protein, and decided to pursue this topic. In addition to 
its canonical role in isoprenoid synthesis in glycosomes, TcMVK is secreted and may be involved in the modulation of cell signaling required for $T$. cruzi invasion of host cells. To date, no Trypanosoma enzymes have been registered in databases as moonlighting proteins. In 2016, a seven-node module (composed of nine genes) that emulates the dynamics of the parasite life cycle was identified using a systems biology approach (Carrea and Diambra, 2016). This module reproduces many important dynamic features observed in the life cycle of $T$. cruzi. In a recent Commentary, the same authors described their findings of unexpected functions in five genes that encode enzymes involved in metabolic pathways: a hexokinase, a d-1-pyrroline-5-carboxylate dehydrogenase, a quinone oxidoreductase, a glutamate dehydrogenase, and a peptidyl-prolyl cis-trans isomerase (Carrea and Diambra, 2017). The authors propose that these metabolic genes encode proteins with other regulatory functions distinct from their original ones, and that the enzymes should therefore be considered moonlighting proteins.

In 2015, Amblee and Jeffery performed proteomics studies that identified intracellular proteins frequently found on the cell surface, some of which displayed distinctive functions in that location and were therefore considered to be moonlighting proteins (Amblee and Jeffery, 2015). The authors suggested that these particular intracellular proteins had adopted certain biophysical features enabling them to perform a second function on the cell surface, but concluded that the moonlighting proteins had physical characteristics typical of intracellular proteins. This is also the case for TcMVK (Ferreira et al., 2016) and many other intracellular proteins that are found on the surface of pathogens and may have moonlighting activity (Gómez-Arreaza et al., 2014; Karkowska-Kuleta and Kozik, 2014).

\section{MOONLIGHTING PROTEINS AND CONTRADICTORY DEFINITIONS}

In my opinion, a precise definition of "moonlighting proteins" remains to be finalized. Several prerequisites need to be considered before deposition to the MoonProt database. In brief, a moonlighting protein is a single protein that displays multiple functions; however, the additional function(s) should be clearly demonstrated using mutagenic methods to impair the first function; i.e., the various functions should be shown to be mutually exclusive (Mani et al., 2014).

With this consideration in mind, I noticed two seemingly contradictory examples that have been deposited in MoonProt: (i) GAPDH of Trichomonas vaginalis was shown by (Lama et al., 2009) to perform its well-documented catalytic functions and also a second fibronectin-binding function; however, the authors did not perform mutagenic studies to demonstrate that the binding function does not depend on the catalytic residues. (ii) Nair et al. (2006) showed that hormone-induced downregulation of luteinizing hormone receptor (LHR) in human ovary granulosa cells is post-transcriptionally regulated by an mRNA binding protein, which was termed LHR-mRNA binding protein (LRBP). LRBP was subsequently shown to be identical to a MVK from rat, and to bind to the coding region of LHR mRNA, thereby suppressing its translation and resulting in degradation of the ribonucleoprotein complex. In a follow-up 2008 study of rat MVK using mutagenesis and crystallography, these authors demonstrated that Ser146, Glu193, Asp204, and Lys13 are essential for MVK binding to mRNA and also for catalytic function of the enzyme, indicating that binding of MVK to LHR mRNA in rats requires an intact active site to suppress translation (Nair et al., 2008).

In case (ii) above, the catalytic residues are necessary for the second function, which seemingly contradicts the MoonProt requisites for moonlighting proteins. On the other hand, the enzyme (MVK) had a second, unexpected function, indicating that it can be considered a moonlighting protein. The definition used for the MultitaskProtDB database is broader, i.e., moonlighting proteins are defined simply as "multitasking" proteins; however, the above proteins are not included in the database. In my opinion, both of the above-described proteins should be considered as moonlighting proteins despite the contradictory initial definitions.

In view of the inconsistency of the definitions of moonlighting proteins used for the two databases, I would like to propose a more precise definition: moonlighting proteins are proteins, typically long-known and well-studied enzymes, found to display one or more additional unexpected or unusual biological functions that were not previously anticipated on the basis of the conserved domains and-or motifs in their primary structure, or three-dimensional structure.

Our study demonstrates that TcMVK participates in parasite internalization, a process unrelated to its expected function. It can be secreted, is found on the parasite cell surface, and adheres to the host cell. Given that TcMVK adheres to the host membrane, it presumably binds to some membrane receptor to trigger yet-unidentified pathway(s) within the host.

According to Jeffery's definition (Jeffery, 1999), a protein can be classified as a moonlighting protein if it performs distinct functions depending on whether it is located inside or outside the cell, or in different cellular compartments.

I therefore suggest that the trypanosomatid protein TcMVK be considered a true moonlighting protein and be formally added to both moonlighting databases.

\section{CONCLUSION}

The findings described above and the unexpected behavior of TcMVK strongly indicate that this kinase is a moonlighting protein (enzyme). On one hand, it is a conserved enzyme that is compartmentalized and plays a role in isoprenoid synthesis in glycosomes; on the other hand, it has a second, unexpected function: it is secreted, adheres to host cells, and likely participates in modulation of host cell signaling, leading to T. cruzi invasion of host cells. TcMVK may thus be an important modulator of $T$. cruzi invasion and a potential target in development of new drugs for treatment of Chagas' disease. This is a novel feature for a T. cruzi enzyme. These observations 
raise various questions regarding how moonlighting proteins evolved, and the selective advantages of having a single protein that performs distinctive multiple functions in an intracellular parasite.

\section{AUTHOR CONTRIBUTIONS}

The author confirms being the sole contributor of this work and approved it for publication.

\section{REFERENCES}

Amblee, V., and Jeffery, C. J. (2015). Physical features of intracellular proteins that moonlight on the cell surface. PLOS ONE 10:e0130575. doi: 10.1371/journal.pone.0130575

Baum, J., Richard, D., Healer, J., Rug, M., Krnajski, Z., Gilberger, T. W., et al. (2006). A conserved molecular motor drives cell invasion and gliding motility across malaria life cycle stages and other apicomplexan parasites. J. Biol. Chem. 281, 5197-5208. doi: 10.1074/jbc.M509 807200

Bhowmick, I. P., Kumar, N., Sharma, S., and Coppens, I., Jarori, G. K. (2009). Plasmodium falciparum enolase, stage-specific expression and sub-cellular localization. Malar. J. 8:179. doi: 10.1186/1475-2875$8-179$

Bosch, J., Buscaglia, C. A., Krumm, B., Ingason, B. P., Lucas, R., Roach, C., et al. (2007). Aldolase provides an unusual binding site for thrombospondinrelated anonymous protein in the invasion machinery of the malaria parasite. Proc. Natl. Acad. Sci. U.S.A. 104, 7015-7020. doi: 10.1073/pnas. 0605301104

Buscaglia, C. A., Coppens, I., Hol, W. G., and Nussenzweig, V. (2003). Sites of interaction between aldolase and thrombospondin-related anonymous protein in plasmodium. Mol. Biol. Cell. 14, 4947-4957. doi: 10.1091/mbc.E0306-0355

Campbell, R. M., and Scanes, C. G. (1995). Endocrine peptides "moonlighting" as immune modulators, roles for somatostatin and GH-releasing factor. J. Endocrinol. 147, 383-396. doi: 10.1677/joe.0. 1470383

Carrea, A., and Diambra, L. (2016). Systems biology approach to model the life cycle of Trypanosoma cruzi. PLoS ONE 11:e0146947. doi: 10.1371/journal.pone.0146947

Carrea, A., and Diambra, L. (2017). Commentary, systems biology approach to model the life cycle of Trypanosoma cruzi. Front. Cell Infect Microbiol. 7:1. doi: $10.3389 /$ fcimb.2017.00001

Carrero-Lerida, J., Perez-Moreno, G., Castillo-Acosta, V. M., Ruiz-Perez, L. M., and Gonzalez-Pacanowska, D. (2009). Intracellular location of the early steps of the isoprenoid biosynthetic pathway in the Trypanosomatids Leishmania major and Trypanosoma brucei. Int. J. Parasitol. 39, 307-314. doi: 10.1016/j.ijpara.2008.08.012

Collingridge, P. W., Brown, R. W., and Ginger, M. L. (2010). Moonlighting enzymes in parasitic protozoa. Parasitology 137, 1467-1475. doi: $10.1017 /$ S0031182010000259

Erez, A., and DeBerardinis, R. J. (2015). Metabolic dysregulation in monogenic disorders and cancer - finding method in madness. Nat. Rev. Cancer 15, 440-448. doi: $10.1038 / \mathrm{nrc} 3949$

Ferreira, É. R., Bonfim-Melo, A., Mortara, R. A., and Bahia, D. (2012). Trypanosoma cruzi extracellular amastigotes and host cell signaling, more pieces to the puzzle. Front. Immunol. 3:363. doi: 10.3389/fimmu.2012. 00363

Ferreira, É. R., Horjales, E., Bonfim-Melo, A., Cortez, C., da Silva, C. V., De Groote, M., et al. (2016). Unique behavior of Trypanosoma cruzi mevalonate kinase, a conserved glycosomal enzyme involved in host cell invasion and signaling. Sci. Rep. 6:24610. doi: 10.1038/srep24610

Gómez-Arreaza, A., Acosta, H., Qui-ones, W., Concepción, J. L., Michels, P. A., and Avilán, L. (2014). Extracellular functions of glycolytic enzymes of

\section{FUNDING}

I thank the support of FAPEMIG (APQ-00474-13, PPM-0060416). DB is a recipient of CNPq Productivity Scholarship.

\section{ACKNOWLEDGMENTS}

I am grateful to Colin Bowles and Dr. S. Anderson for English editing of the manuscript.

parasites: unpredicted use of ancient proteins. Mol. Biochem. Parasitol. 193, 75-81. doi: 10.1016/j.molbiopara.2014.02.005

Henderson, B., and Pockley, A. G. (2010). Molecular chaperones and protein-folding catalysts as intercellular signaling regulators in immunity and inflammation. J. Leukoc. Biol. 88, 445-462. doi: 10.1189/jlb. 1209779

Henderson, B., and Martin, A. (2011). Bacterial moonlighting proteins and bacterial virulence. Curr. Top. Microbiol. Immunol. 15, 440-448. doi: 10.1007/82_2011_188

Huberts, D. H., and van der Klei, I. J. (2010). Moonlighting proteins, an intriguing mode of multitasking. Biochim. Biophys. Acta 1803, 520-525. doi: 10.1016/j.bbamcr.2010.01.022

Jeffery, C. J. (1999). Moonlighting proteins. Trends Biochem. Sci. 24, 8-11. doi: 10.1016/S0968-0004(98)01335-8

Jewett, T. J., and Sibley, L. D. (2003). Aldolase forms a bridge between cell surface adhesins and the actin cytoskeleton in apicomplexan parasites. Mol. Cell. 11, 885-894. doi: 10.1016/S1097-2765(03)00113-8

Karkowska-Kuleta, J., and Kozik, A. (2014). Moonlighting proteins as virulence factors of pathogenic fungi, parasitic protozoa and multicellular parasites. Mol. Oral Microbiol. 29, 270-283. doi: 10.1111/omi. 12078

Krishnamurthy, G., Vikram, R., Singh, S. B., Patel, N., Agarwal, S., Mukhopadhyay, G., et al. (2005). Hemoglobin receptor in Leishmania is a hexokinase located in the flagellar pocket. J. Biol. Chem. 280, 5884-5891. doi: $10.1074 /$ jbc.M411845200

Lama, A., Kucknoor, A., Mundodi, V., and Alderete, J. F. (2009). Glyceraldehyde3-phosphate dehydrogenase is a surface-associated, fibronectin-binding protein of Trichomonas vaginalis. Infect. Immun. 77, 2703-2711. doi: 10.1128/IAI.00157-09

Mani, M., Chen, C., Amblee, V., Liu, H., Mathur, T., Zwicke, G., et al. (2014). MoonProt: a database for proteins that are known to moonlight. Nucleic Acids Res. 43, D277-D282. doi: 10.1093/nar/ gku954

Nair, A. K., Peegel, H., and Menon, K. M. J. (2006). The role of luteinizing hormone/human chorionic gonadotropin receptor-specific mRNA binding protein in regulating receptor expression in human ovarian granulosa cells. J. Clin. Endocrinol. Metab. 91, 2239-2243. doi: 10.1210/jc.20052739

Nair, A. K., Young, M. A., and Menon, K. M. (2008). Regulation of luteinizing hormone receptor mRNA expression by mevalonate kinase-role of the catalytic center in mRNA recognition. FEBS J. 275, 3397-3407. doi: $10.1111 /$ j.1742-4658.2008.06490.x

Quinones, W., Pena, P., Domingo-Sananes, M., Caceres, A., Michels, P. A., Avilan, L., et al. (2007). Leishmania mexicana, molecular cloning and characterization of enolase. Exp. Parasitol. 116, 241-251. doi: 10.1016/j.exppara.2007. 01.008

Vanegas, G., Quinones, W., Carrasco-Lopez, C., Concepcion, J. L., Albericio, F., and Avilan, L. (2007). Enolase as a plasminogen binding protein in Leishmania mexicana. Parasitol. Res. 101, 1511-1516. doi: 10.1007/s00436-0070668-7

Watanabe Costa, R., da Silveira, J. F., and Bahia, D. (2016). Interactions between Trypanosoma cruzi secreted proteins and host cell signaling pathways. Front. Microbiol. 7:388. doi: 10.3389/fmicb.2016. 00388 
Weaver, D. T. (1998). Telomeres, moonlighting by DNA repair proteins. Curr. Biol. 8, R492-R494. doi: 10.1016/S0960-9822(98)70315-X

Zhang, K., and Rathod, P. K. (2002). Divergent regulation of dihydrofolate reductase between malaria parasite and human host. Science 296, 545-547. doi: $10.1126 /$ science. 1068274

Conflict of Interest Statement: The author declares that the research was conducted in the absence of any commercial or financial relationships that could be construed as a potential conflict of interest.
The reviewer NR and handling Editor declared their shared affiliation.

Copyright (c) 2017 Bahia. This is an open-access article distributed under the terms of the Creative Commons Attribution License (CC BY). The use, distribution or reproduction in other forums is permitted, provided the original author(s) or licensor are credited and that the original publication in this journal is cited, in accordance with accepted academic practice. No use, distribution or reproduction is permitted which does not comply with these terms. 